\section{Mechanism of Strong Effects of Stall Suppression by an Air Separator Investigated by Internal Flow Measurements in an Axial Flow Fan*}

\author{
Nobuyuki YAMAGUCHI**, Masayuki OGATA** and Shinki TANAKA*** \\ ${ }^{* *}$ Meisei University, \\ 2-1-1, Hodokubo, Hino-shi, Tokyo, Japan \\ E-mail: yamagucn@me.meisei-u.ac.jp \\ ***TOKIKO Technology, Ltd., \\ 3-9-27, Tsurumichuo, Tsurumi-ku, Yokohama-shi, Japan
}

\begin{abstract}
Radial-vaned air separators produce strong stall improvement effect in axial flow fans. Changes in the fan internal flow patterns achieved by the device are confirmed by Pitot traverse measurements. For the air separator condition, with a decrease in the fan flow rate the meridional streamline inclinations through the rotor blades have become increasingly steep and the circumferential velocities increase toward the tip region, in contrast to relatively little changes in the behaviors of the streamlines and flow patterns in the solid wall condition. It is suggested that the stall suppression is achieved by a combination of the following effects of the air separator; (1) suction of casing boundary layers and elimination of embryos of stall, (2) separation of reversed flow from the main flow, (3) induction of the flow toward the casing wall and strengthening of meridional streamline inclination, thus keeping the Euler head increasing, and (4) reinforcement of axi-symmetric flow structure. Appearance of these effects in the descending order is considered to achieve the great stall suppression effect that would be difficult by other types of stall prevention devices.
\end{abstract}

Key words: Fluidmachinery, Axial Flow Fan, Stall, Stall Improvement, Air Separator, Internal Flow

\section{Foreword}

As concerns for global environment and energy resources increase, higher efficiencies have been demanded for both energy generations and consumptions. Some of the demands have been directed toward turbomachines such as gas turbines, jet engines, compressors and fans, etc. If stalling characteristics of these machines are improved, the wider range of high-efficiency near the stalling point would provide not only safer operations but also much improvement in the working efficiencies of the power plants and the industrial plants.

With the above in mind, the authors ${ }^{(1)-(4)}$ have been concerned with improvement of the stall characteristics of axial flow compressors and fans, particularly by using air separator devices. The air separator device discussed here is constructed of radial-vanes with leading edges that scoop directly the flow near the rotor tip, as described in Section 3. The flow de-swirled through the vanes turns axially and joins the main flow at the device exit upstream. The system has provided much better stall prevention effect compared with other similar devices. For example, in the best situation, the air separator could attain nearly $100 \%$ of the stalling flow improvement ${ }^{(1),(3)}$, compared with a maximum of 20 to $40 \%$ by
*Received 30 Nov., 2010 (No. T2-10-0289)
Japanese Original : Trans. Jpn. Soc. Mech.
Eng., Vol.76, No.771, B (2010),
pp.1727-1735 (Received 12 Apr., 2010)
[DOI: 10.1299/jst.6.202]

Copyright $\odot 2011$ by JSME 
casing treatments ${ }^{(5)}$. In addition to the significant stall improvement effect, the same kind of the air separator device employed here was relatively unaffected by the blade tip speed, and so, it was stable up to higher rotor speeds ${ }^{(6)}$.

The cause for the high level of effectiveness achieved by not only the air separator discussed here but also other favorable ones (for example, the air separators cited in the appendices of Refs. (1) and (3)) has been a long-year question to the authors. So, the authors conducted surveys on the internal flow behaviors of both the solid wall condition and the air separator condition to discover a clue ${ }^{(7)-(9)}$. This report summarizes the results and the probable cause of the positive effect of the air separator.

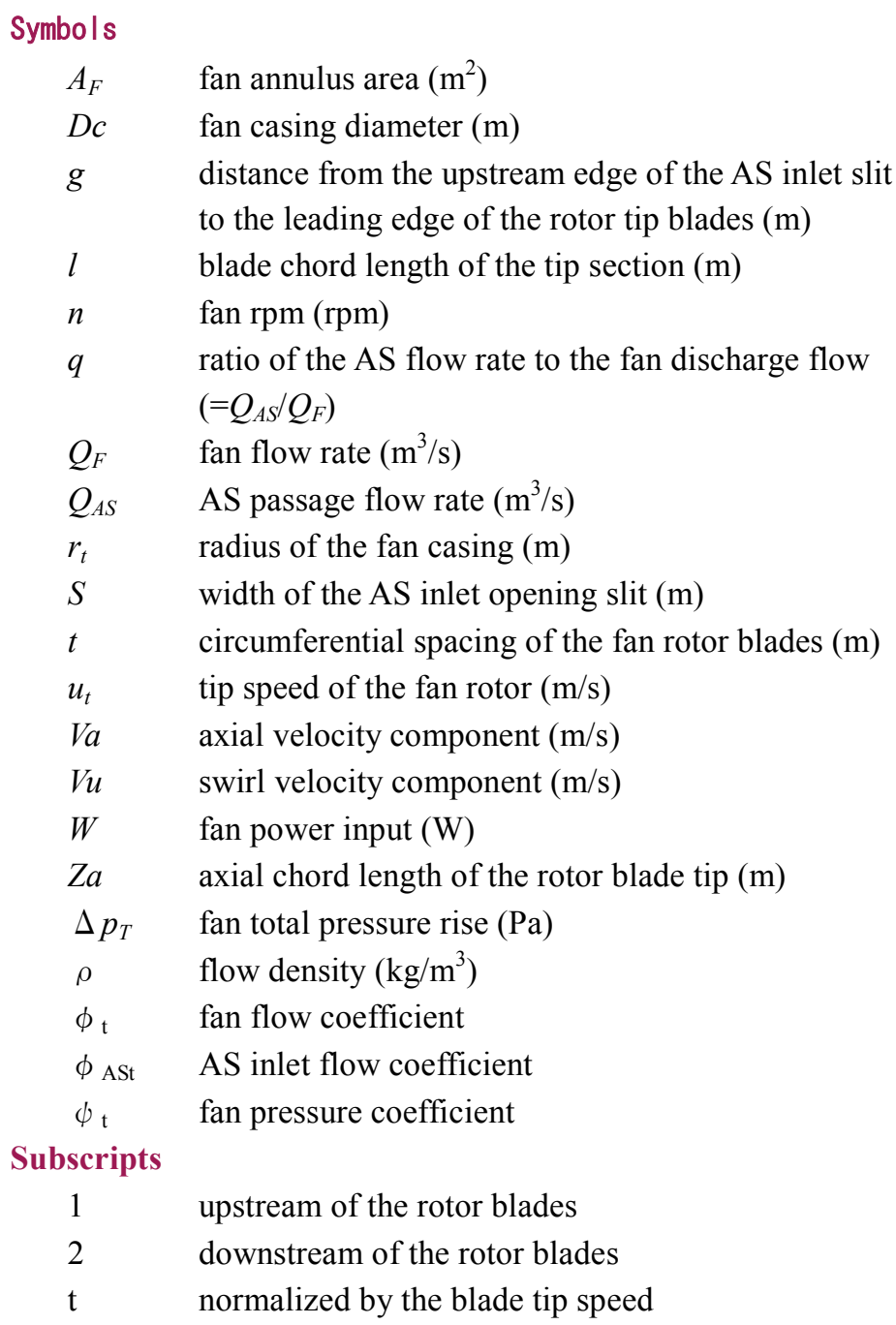

\section{Outline of the Measurements}

The experimental fan is a commercially available one modified for an air separator device (hereafter referred to as "AS"). The fan dimensions are given in Table 1 and the construction is shown in Fig. 1, where "AS" is the air separator device and "OGV" is the exit guide vanes. The geometry of the rotor blade shown in Fig. 2 includes the following: blade chord lengths, camber angles and stagger angles in the plane perpendicular to the blade height direction versus radial position. The blade data distribution was measured along the blade height for only one blade, and typical data on blade chord length and angles were measured for all blades at the three radial positions of hub, mean, and tip. The data 
scatter in the blade geometry was $+-2 \mathrm{~mm}$ for the lengths and +- 5 degrees for the angles. The geometry data show, although the stagger angle increases monotonically from the root to the tip, the camber angle shows an unusual trend compared with ordinary axial-fan blades in that the maximum blade camber angle lies at a height approximately $40 \mathrm{~mm}$ from the root. The blades were manufactured by twisting a single sheet of metal plate. The simple manufacturing procedure might be responsible for the blade configuration, and the blade configuration could have influenced the internal flow conditions and the overall performances. This potential influence is not considered a problem because this study evaluated only the comparative differences in the flow conditions in the same fan with and without the AS device to clarify the mechanism of the AS effect. Table 2 lists the average data at the blade tip and root.

The fan working conditions were changed by throttling the

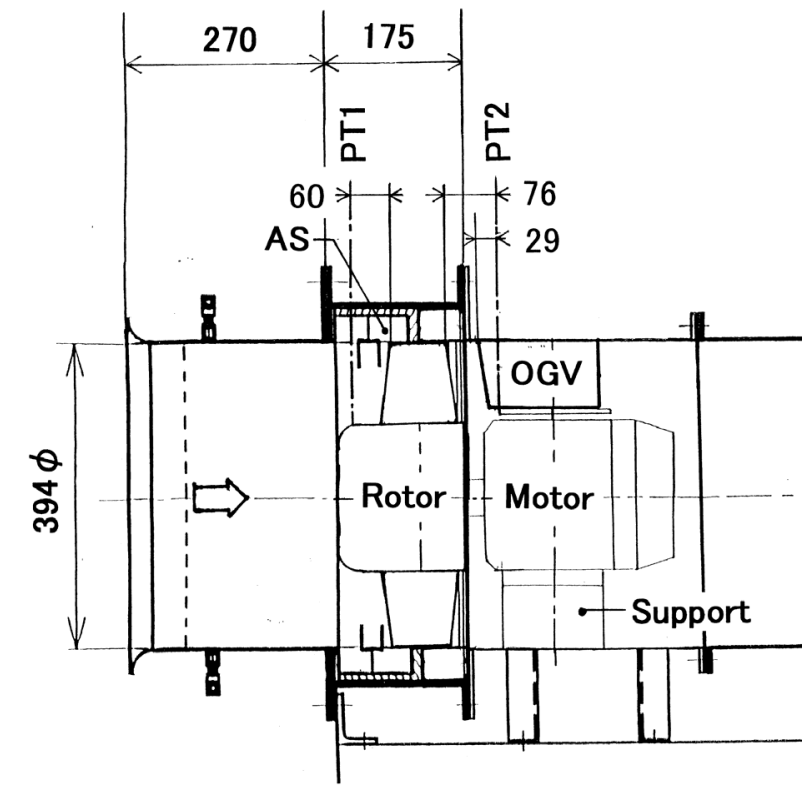

Fig. 1 Axial flow fan for test and locations of Pitot traverse measurements, where AS: air separator device, OGV: exit guide vanes.

exit valve. Then the fan flow rate was measured by a Venturi-tube differential pressure, the fan pressure rise by manometers, the fan power input by an electric power meter, and the fan speed by a tachometer. Measurements of the local internal flow, including pressures, flow velocities and flow angles, were conducted by traversing a small three-hole yawmeter.

The Reynolds number of the blade based on the blade tip speed and the blade chord was approximately $3.5 \times 10^{5}$.

The accuracy of the measurements is described later in Section 11.

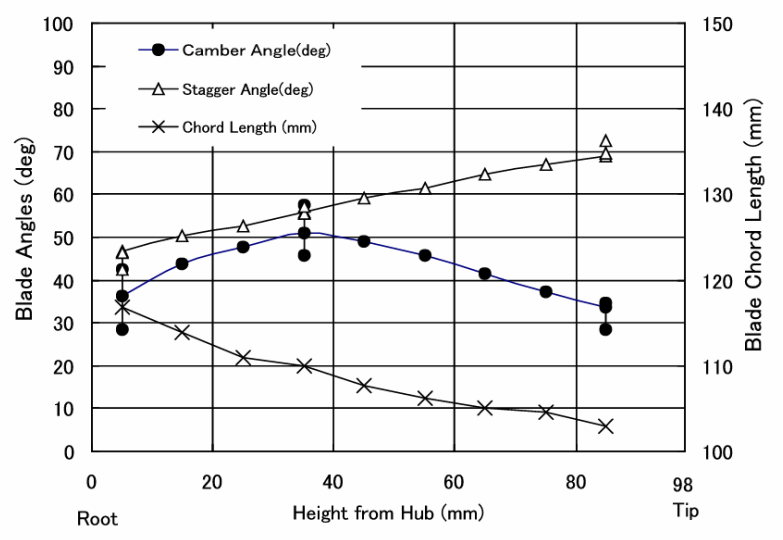

Fig. 2 Measured blade parameters in the straight planes perpendicular to the blade centerline

Table 1 Dimensions of the fan for test

\begin{tabular}{|l|c|}
\hline Casing diameter & $394 \mathrm{~mm}$ \\
\hline Tip diameter / Hub diameter & $390 \mathrm{~mm} / 194 \mathrm{~mm}$ \\
\hline Speed & $2600 \mathrm{rpm}$ \\
\hline Motor power & $1.5 \mathrm{~kW}$ \\
\hline Pressure rise / Flow rate & $0.20 \mathrm{kPa} / 100 \mathrm{~m}^{3} / \mathrm{min}$ \\
\hline
\end{tabular}


Table 2 Average geometric dimensions of the rotor blades measured in a straight plane perpendicular to the blade height direction

\begin{tabular}{|l|c|c|}
\hline & Tip & Root \\
\hline Number of rotor blades & \multicolumn{2}{|c|}{6} \\
\hline Chord length $l(\mathrm{~mm})$ & 103 & 118 \\
\hline Blade spacing $t(\mathrm{~mm})$ & 204 & 102 \\
\hline Pitch chord ratio $t / l$ & 1.98 & 0.86 \\
\hline Stagger angle $\xi(\mathrm{deg})$ & 71 & 43 \\
\hline Camber angle $\varphi(\mathrm{deg})$ & 25 & 32 \\
\hline
\end{tabular}

\section{Conditions for the Air Separator and the Solid Wall}

The arrangement relative to the AS device used in the investigation is shown in Fig. 3 and Fig. 4 shows the de-swirl vanes in the AS device (shaded part in Fig. 3). In Fig. 3, "S" is the axial width of the AS inlet slit and " $g$ " is the distance between the leading edge of the blade tip and the upstream end of the AS inlet slit. This investigation used the following relative value, which has been found to be in the optimum range in the preceding study ${ }^{(1),(3)}$.

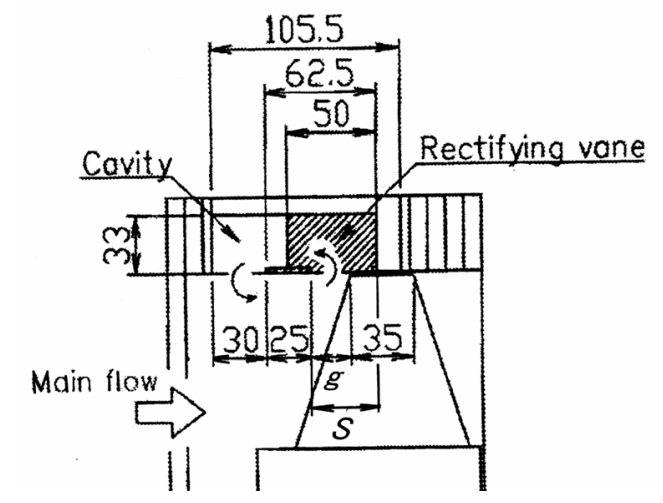

Fig. 3 Layout of the air separator relative to the fan blades

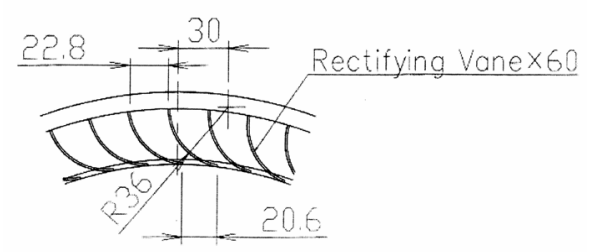

Fig. 4 Configuration of the air separator de-swirl vanes

\section{Characteristic Parameters}

The overall performances are expressed by the following dimensionless quantities normalized by blade tip speed $u_{t}$.

$$
\begin{array}{ll}
\text { Fan flow coefficient } & \phi_{t}=Q /\left(u_{t} A_{F}\right) \\
\text { Fan total pressure coefficient } & \psi_{T 2-1}=\Delta p_{T 2-1} /\left(0.5 \rho u_{t}^{2}\right)
\end{array}
$$

The flow velocities upstream and downstream of the rotor row are normalized also by blade tip speed $u_{t}$.

$$
\begin{array}{ll}
\text { Axial velocity coefficient } & V_{a 1} / u_{t}, V_{a 2} / u_{t} \\
\text { Swirl velocity coefficient } & V_{u 1} / u_{t}, V_{u 2} / u_{t}
\end{array}
$$

Here, $V a$ : axial velocity, $V u$ : swirl velocity, $u_{\mathrm{t}}$ : blade tip speed, $\triangle P_{T 2-1}$ :total pressure rise from upstream to downstream of the rotor blade row, $\rho$ : air density, $Q$ : fan flow rate, $A_{F}$ :fan annulus area. Subscripts 1 and 2 represent the upstream and downstream locations of the rotor blade row, respectively. 


\section{Flow Conditions}

The overall performances of the AS condition and the SW one are compared in Fig. 5. In the SW condition, the total-to-total pressure coefficients $\phi_{\mathrm{T} 2-1}$ reaches the peak around flow coefficient $\phi_{\mathrm{t}}=0.263$, below which $\psi_{\text {T2-1 }}$ lowers. The point is named as SW stalling point. Below $\phi_{t}$ of 0.16 , further throttling caused flow reversal up to the suction duct inlet, which was observed by a tuft grid there. The flow reversal was found to affect the fan upstream wall pressure and the indicated fan pressure rise. Therefore, for comparison with the AS performances, the SW performance in Fig. 5 is valid above $\phi_{t}$ of 0.16 , and the zone for $\phi_{\mathrm{t}}$ below 0.16 is included only for reference.

The AS performance shows negative slope almost everywhere, i.e., no stall occurs to the near-shut-off condition.

The measurements of the internal flows were conducted for nine representative working

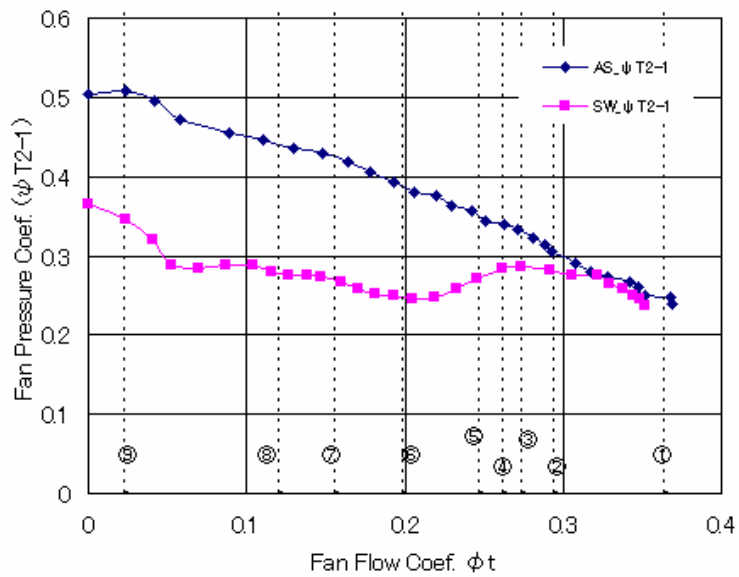

Fig. 5 Fan performances for the AS and the SW conditions, indicating points of the measurements of the fan internal flows

Table 3 Measuring points of the internal flows

\begin{tabular}{|c|l|c|}
\hline & Condition & $\phi \mathrm{t}$ \\
\hline (1) & Maximum flow & 0.364 \\
\hline (2) & In SW sound condition & 0.295 \\
\hline (3) & Before SW stalling point & 0.275 \\
\hline (4) & SW stalling point & 0.263 \\
\hline (5) & After SW stalling & 0.247 \\
\hline (6) & In SW stall & 0.200 \\
\hline (7) & $\begin{array}{l}\text { SW flow reversal up to } \\
\text { the suction duct Inlet }\end{array}$ & 0.157 \\
\hline (8) & In SW deep stall & 0.121 \\
\hline (9) & Near-shut-off condition & 0.023 \\
\hline
\end{tabular}
conditions, indicated as (1) (9) in Fig. 5. The working conditions are given in Table 3 . Conditions (1) (6) were measured for both the SW and the AS, and (7) (9) were measured only for the AS condition, because the flow fluctuations and disturbances in the SW condition were too great for accurate measurements of the average flow.

The three-hole yawmeter is a cobra-type device having a tiny head with pressure holes of $0.5 \mathrm{~mm}$ in diameter, and the head size of thickness $1 \mathrm{~mm}$ and width $3 \mathrm{~mm}$. The yawmeter was inserted through the fan casing wall perpendicular to the fan axis for nearly two-dimensional or axi-symmetric flow, with less attention to radial flows. The authors considered that the procedure could clarify the major qualitative features of the changes in the flow behaviors caused by the AS application, although the procedure did not have sufficiently high accuracy in the highly three-dimensional flow field.

The axial locations for the internal measurements are shown in Fig. 1: Location PT1 is $60 \mathrm{~mm}$ upstream from the leading edge of the rotor tip blade section and Location PT2 is 76 $\mathrm{mm}$ downstream from the trailing edge of the rotor tip blade section. Location PT1 passes through the AS exit slit. Location PT2 lies between the exit guide vanes, $29 \mathrm{~mm}$ downstream from the leading edges at the casing wall. The yawmeter insertion hole is only one in number in the total circumference for each of Locations PT1 and PT2. 


\section{Internal Flow Pattern for the Solid Wall Condition}

Figures 6-9 show the flow patterns for the SW conditions. "SW_X" in the figures mean the number of the SW working conditions, respectively, given in Fig. 5 and Table 3.

The upstream axial velocities in Fig. 6 increases slightly toward the hub, which shows the effect of the round head of the fan hub. Along the casing wall exists a relatively thick boundary layer approximately $20 \mathrm{~mm}$ in width. The thicker boundary layer developed possibly because the continued long suction duct wall has the same diameter. The upstream swirl velocities in Fig. 7 are nearly zero. The scatter suggests random errors in the measurement of an order of approximately $2 \%$ maximum of the fan tip speed.

The downstream axial velocities in Fig. 8 show gradual inverted S-shaped distributions. For changes in the fan flow rates, the axial velocities do not appear to show distinctive changes in the S-shaped distributions, accompanying only changes in the average levels. The local increase in the axial velocities seen in the tip region in the radius ratio above 0.9 is supposed to represent the local increase in the blade work due to the thick inflow boundary layer.

The downstream swirl velocities in Fig. 9 show small increases in the tip region and have small peaks around radius ratio $0.85-0.9$, showing flat letter "3"- shapes as a whole, except for the maximum flow (1) where the pattern shows a concave shape. For the wide range of the fan flow rate, the distributions change as a whole in a similar manner. Also, the stalled conditions, (5) and (6), show a similar tendency.

The particular behaviors of the downstream velocities for the SW condition described above appear somewhat different from ordinary downstream velocities, which tend to have nearly uniform or monotonically changing axial velocity distributions and monotonically decreasing swirl velocity distributions in the radial direction except for the boundary layer regions close to the casing wall and the hub wall. One of the possible causes for these different behaviors could be a change in the radial equilibrium owing to the singular distribution of the blade parameters shown in Fig.2. In addition, the increased swirl velocities owing to the lower inflow axial velocities in the thick boundary layer and the related secondary passage vortex effects could be an influence.

\section{Internal Flow Pattern for the Air Separator Condition}

Figures 10-13 show the internal flow patterns for the AS device applied to the above fan. "AS_X" in the figures mean the number of the AS working conditions, respectively, given in Fig. 5 and Table 3. The following characteristic changes from the SW conditions were observed.

The upstream axial velocities in Fig. 10 show a tendency similar to those for the SW condition in Fig. 6. The upstream swirl velocities in Fig. 11 are essentially zero, but some have a swirling zone toward the tip in the flow range corresponding to the flow range from the SW stall (6) to the near-shut-off condition (9). In this flow range the flow rate through the AS passage is considered to be too large to be completely de-swirled.

For decreases in the fan flow rate, the downstream axial velocities in Fig. 12 generally tend to increase toward the tip region and accompany decreases toward the inner region below 0.87 of the radius ratio. In other words, the flow tends to gather toward the blade tip. Finally, in condition (8) corresponding to the SW deeply stalled condition and the nearshut-off condition (9, the axial velocities continue to increase monotonically from the hub to the tip, except in the casing wall boundary layer.

It is noteworthy that, in the changes in the fan flow rate, the distribution pattern of the downstream axial velocities changes itself in the radial direction. The behavior is contradictory to those in the SW conditions, where the pattern shows no apparent changes, except for changes mainly in the average level. The marked difference is clear in Figs. 12 and 8 . 


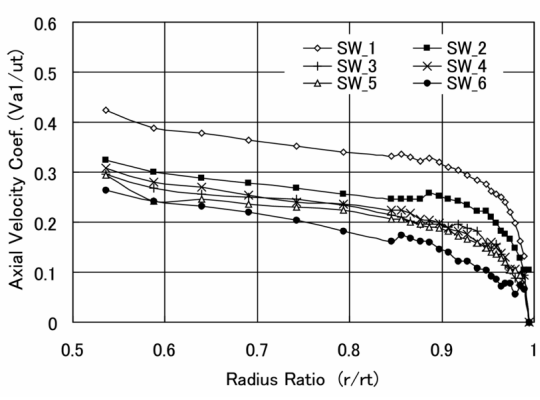

Fig. 6 Distributions of the axial velocity upstream of the rotor for the SW condition

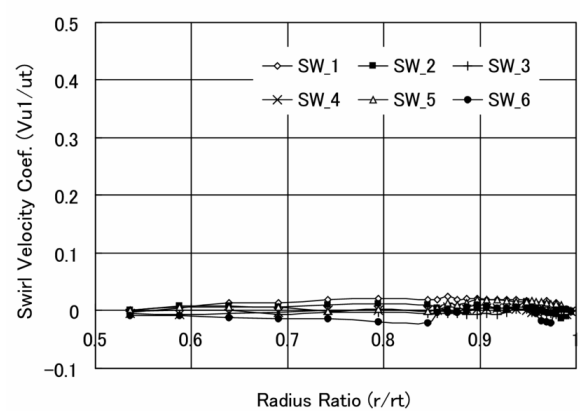

Fig. 7 Distributions of the swirl velocity upstream of the rotor for the SW condition

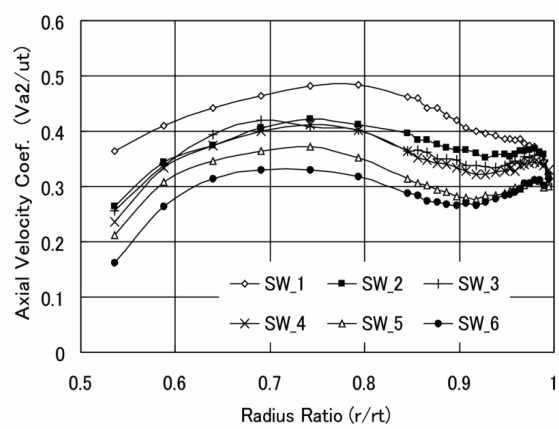

Fig. 8 Distributions of the axial velocity downstream of the rotor for the SW condition

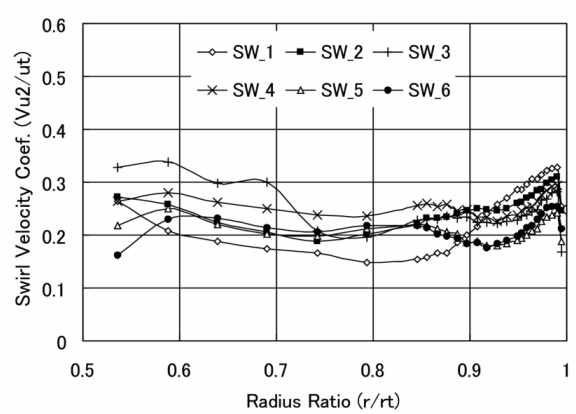

Fig. 9 Distributions of the swirl velocity downstream of the rotor for the SW condition

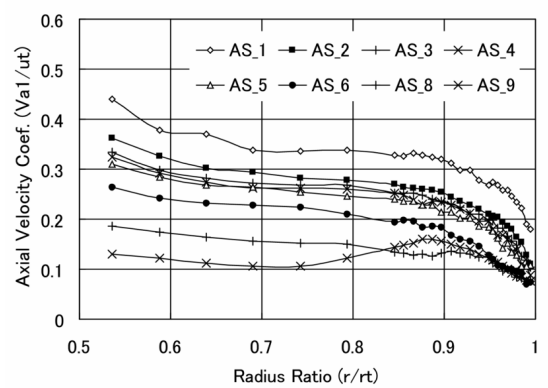

Fig. 10 Distributions of the axial velocity upstream of the rotor for the AS condition

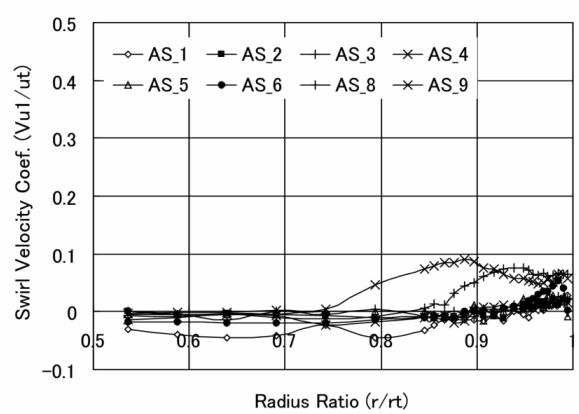

Fig. 11 Distributions of the swirl velocity upstream of the rotor for the AS condition

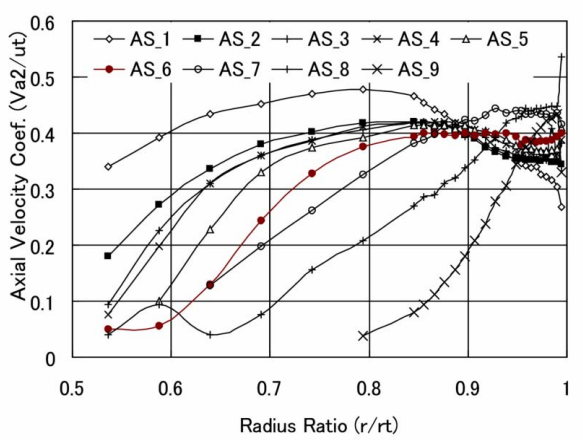

Fig. 12 Distributions of the axial velocity downstream of the rotor for the AS condition

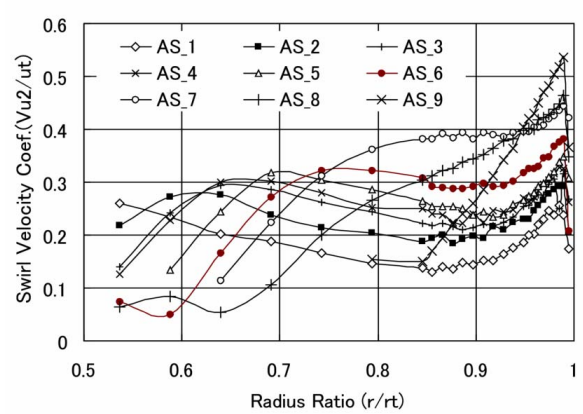

Fig. 13 Distributions of the swirl velocity downstream of the rotor for the AS condition 
The flow in the neighborhood close to the casing wall in the AS condition has a greater velocity and a thinner boundary layer in comparison with those in the SW condition. The near-wall flow could have been activated for the AS condition.

Distributions of the downstream swirl velocities in Fig. 13 show inverted S-shapes increasing toward the tip and decreasing toward the hub, but also show a plateau and mild peak in between. With the reduction in the fan flow rate, the velocities tend to increase more and more toward the tip except in the very thin casing boundary layer. The decreasing tendency of the swirl velocities toward the hub corresponds to that of the axial velocities shown at the same place in Fig. 13. Some of the measurements downstream of the rotor row for AS_9, i.e., near-shut-off condition, were not successful near the hub region because of the very low air velocities in the situation of the extremely tip-concentrated flow.

Another result suggesting the flow patterns in the AS condition is shown in Fig. 14, which plots the distributions of the static pressures along the casing wall measured through approximately axially arranged pressure taps from near to the rotor trailing edge to upstream of the exit guide vanes. Location $x / Z a=0$ is at the

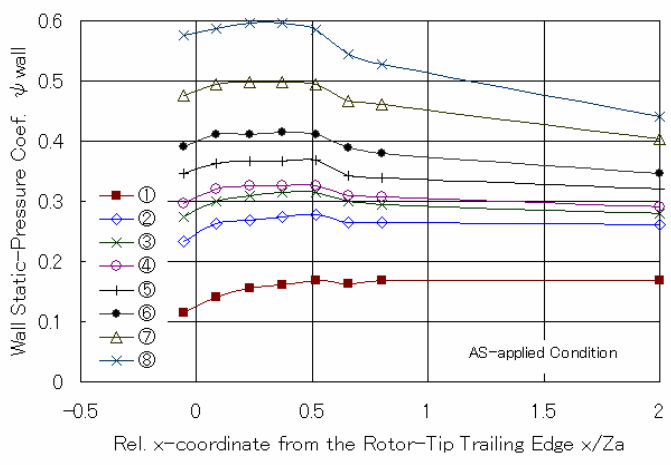

Fig. 14 Axial distribution of the wall pressures from the trailing-edge plane of the rotor-tip to the static pressure location for the AS condition trailing edge of the rotor blade tip and $x / Z a=2$ is near the rear Pitot traverse position, PT2, where $Z a$ is the axial chord length of the blade tip. The data are as-measured gauge pressures normalized by the rotor tip speed dynamic pressure. Distributions of the wall pressures between locations 0.8 and 2 of $x / Z a$, although not measured, are assumed to be smooth and monotonous because no particular obstructs exist between these two locations.

For larger flow conditions, the pressure distributions are relatively flat. For reduced flows, the distributions tend to increase immediately after the blade trailing edge to approximately $0.6 \mathrm{x} Z a$ and after that they decrease somewhat and become flatter. These results suggest that, in the reduced flow region, strongly inclined meridional streamlines pass through the blade rows and strike the casing wall, where they are forced to turn to be along the wall. This situation suggests that the meridional streamlines have a much greater inclination in the neighborhood close to the blades than do the straight streamlines shown later in Fig. 15, as assumed on the basis of axial velocity distributions at the relatively far locations.

\section{The Meridional Streamlines}

The above observations suggest that the meridional streamlines through the rotor blade row were reinforced to incline radially outward by the AS device. From the experimental data on the axial velocities and the recirculation flow rate through the AS passage, the approximately estimated meridional streamlines are drawn as streamtubes passing respectively $10 \%$ of the sectional integrated flow. For the AS condition, the upstream sectional integrated flow was increased by the recirculation flow through the AS passage. The uppermost streamlines in the AS condition, which are not coincident with the casing wall, reflect the existence of AS recirculation flow joined upstream.

The streamlines incline toward the tip, a tendency enhanced by the decreases in the fan flow rate. The tendency become more clear in the range corresponding to the SW stalled range (4) (8). At the same time, the hub streamtubes expand, indicating a lower downstream velocity and, in the extreme case, possibly, a reversed flow. 
The streamlines are shown as straight lines passing through the corresponding points at sections of PT1 and PT2. The wall pressure behavior observed in Fig. 14 suggests that, in the low flow region, the streamlines could be more steeply inclined toward the casing immediately downstream of the rotor blade row, and after that, they could turn to the axial direction along the wall, passing through the corresponding points.

One of the causes of the enhanced inclination of meridional streamlines could be an effect of the flow passing through the AS, which could accompany and induce the neighboring flow toward the casing wall, in addition to the ordinary blade centrifuging action.

Thus, this enhanced inclination increases the Euler work or pressure rise along the streamlines, which is reflected by the swirl velocities increasing toward the tip, as shown in Fig. 13. The above sequence could be one of the driving forces that keep the fan pressure rising for the decreasing fan flow, i.e., that keep the negative slope in the flow-pressure characteristics of the fan.

In addition to the above consideration, the tip flow structure and the streamline configuration could be controlled by the presence of the vortex ring encasing the blade tip through the AS passage. Furthermore, the swirl velocities increasing toward the tip zone are supposed to reinforce the axi-symmetry of the flow field. Near the hub region, axial velocities tend to drop, compensating for the strengthened inclinations of the tip streamlines, and the swirl velocities also drops correspondingly. In the lower

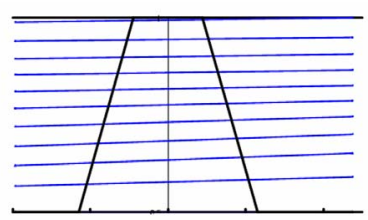

Streamlines for AS_1

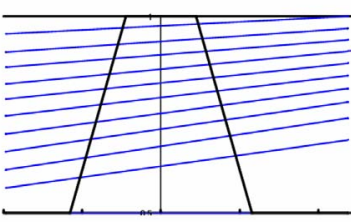

Streamlines for AS_6

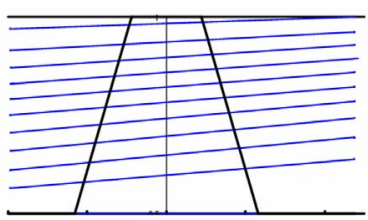

Streamlines for AS 4

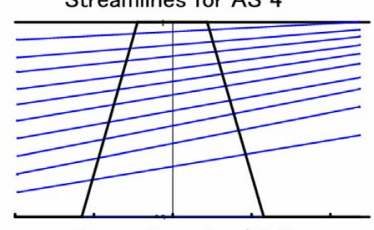

Streamlines for AS_8

Fig. 15 Approximately estimated meridional streamline patterns across the rotor blades for AS conditions 1, 4, 6 and 8 , constructed from the axial velocity distributions (Flow from left to right)

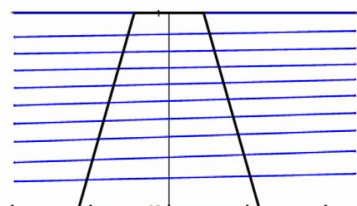

Streamlines for SW_1

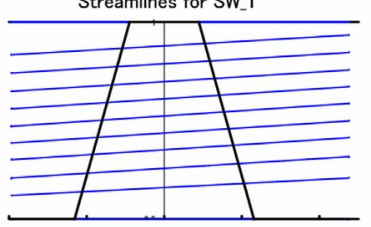

Streamlines for $\mathrm{SW}_{-} 6$

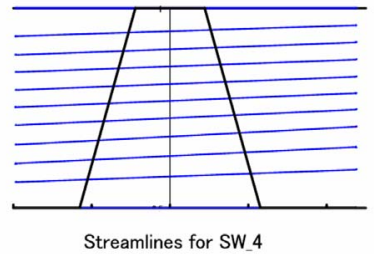

Streamlines for SW 4

Fig. 16 Approximately estimated meridional streamline patterns across the rotor blades for SW conditions 1, 4 and 6 , constructed from the axial velocity distributions (Flow from left to right) fan flow conditions corresponding to the SW stalled region, reversed flow or vortices could have occurred near the hub region.

Combination of the enhanced streamline inclination and the presence of the tip vortex could strengthen the axi-symmetrical structure of the flow field near the blade tip, resulting in the suppression of the circumferential instability, i.e., rotating stall. In fact, hot-wire measurements revealed an occurrence of rotating stalls in the SW stalling conditions, but none in the AS conditions ${ }^{(10)}$.

Figure 16 shows meridional streamlines in the SW conditions estimated similarly to those in the AS condition for comparison. For the changing fan flow rates, the streamlines 
run in a comparatively parallel fashion to the fan axis, which is quite different from the AS situation above.

It is to be noted that the streamlines are quite qualitative, not quantitative, due to numerous experimental limitations.

\section{Flow Rate in the AS Passage}

The flow rates through the AS passage were estimated on the basis of the axial velocities measured in the exit opening of the AS device at PT1 in Fig. 5. The flow forms a wall jet attached along the ceiling of the AS passage. The velocity distribution was integrated to give the AS flow rate $Q_{A S}$. The AS flow ratio $q$ is defined by Eq. (3).

$$
q=Q_{A S} / Q_{F}
$$

Figure 17 shows the distribution of the AS flow ratio $q$ in relation to the fan flow coefficient $\varphi_{\mathrm{t}}$. For the flow range from the maximum flow to the SW stalling corresponding point, the AS flow ratio ranges 2 to $3 \%$. It increases abruptly to approximately $7 \%$ around the SW stalling equivalent point. Such a relatively small extent of the flow ratio enables the stall suppression and the changes in the internal flow conditions over the AS flow range corresponding to the SW mildly stalled range. It is unknown at this time why the marked change in the AS flow ratio seen around the SW stalling-equivalent flow occurs.

In the near-shut-off condition, the AS flow ratio increases to roughly $100 \%$. The AS flow rate amounts to nearly the same as the fan discharge flow rate.

Figure 18 shows the AS inlet flow coefficient, which is the radial inflow velocity through the AS inlet opening slit normalized by the rotor tip speed, defined as follows;

$$
\phi_{A S t}=Q_{A S} /\left(2 \pi r_{t} S u_{t}\right)
$$

The coefficient is 0.01-0.04 except near the shut-off condition.

Below the flow coefficient corresponding to the SW stalling point, the AS passage flow increases spontaneously, and enhances the above stall suppression effects. Thus, the AS device appears to have the ability of passive stall control.

\section{Mechanism of the AS Stall Suppression Effect}

The strong effect achieved by the AS devices could not be explained completely by the descriptions given in the foreword of the preceding papers ${ }^{(1),(3)}$ or by the simple effect of environmental cleaning by the device, described in item (1) below. The above observations suggest that the following effects, which appear in descending order of their effects, are combined and integrated to result in the overall effect. 
(1) Cleaning of the blade working environment Removal by the AS suction effect of low energy fluid, such as the upstream boundary layer flow and blade boundary layer flow, and embryos of reversed flow or rotating stall cells near the tip leading edges could keep a clean blade working environment. It could enable the blade cascade work to its full ability without being deteriorated by the low-energy stall-threatening surrounding flow. The effect is considered to be similar to that of casing treatments ${ }^{(5)}$.

(2) Separation of the reversed flow Separation and isolation of the reversed flow appearing upstream of the blade tips both near and in stalling conditions eliminates direct interference with the main inflow, thus preventing destabilization of the flow. This is the primary purpose of the air separator, as the name suggests.

The above two effects are the mechanisms expected ordinarily for the AS devices. In addition to the above, this study suggests that the following effects play important roles in stall suppression.

(3) Reinforcement of inclinations of the meridional streamlines Flows near the tip region could be strongly forced to head to the casing wall by the accompanying action of the AS suction flow, resulting in greater inclination of the meridional streamlines through the rotor blade row. As the fan flow decreases, the tendency is stronger. The reinforced inclination increases the Euler work or pressure rise along the streamlines, maintaining the fan negative-slope characteristics.

(4) Stabilization of the flow structure by a tip vortex ring The presence of a vortex ring encasing the blade leading-edge tips generated by the AS passage flow and its recirculation flow, along with the blade tip flow, could make the flow field axi-symmetric, thus tending to stabilize the flow structure around the fan tip and to reinforce the streamline inclination.

(5) Enhancement of the axi-symmetry of the fan flow field The AS applied fan respond to the reduction in the fan flow rate by changing the flow pattern radially where both axial and swirl velocities tend to increase toward the tip. This action is considered to enhance the axi-symmetry of the tip flow structure, thus increasing the circumferential stability. The authors suppose that the stability also tends to suppress the rotating stall initiation.

Contrary to the above, the solid wall fan responds to the reduction in the fan flow rate by lowering the average level of axial velocities with little changes in the radial patterns of flow, which is supposed to relatively easily yield circumferential instability, i.e., rotating stall, under some working conditions.

The appendices of Ref. (1) and (3) summarize the AS effects as line segments connecting both SW stalling points and the respective corresponding improved AS stalling points on many fans with various design parameters. The global tendency of the AS improvements indicates that most of the line segments are headed to the approximate value 0.5 of the total-to-static pressure coefficient $\psi_{t T-S}$ and the total-to-total pressure coefficient $\psi_{t T-T}$ normalized by the blade tip speed, at the shut-off condition or the zero flow coefficient $\varphi_{t}=0$. The tendency suggests that a common flow structure related closely with the blade tip region dominates the stall suppression phenomenon. The authors are convinced that the above items (3)-(5) are the phenomena controlling the flow structure.

The effects of stall prevention by other device types, such as casing treatments, appear smaller in comparison with the effects of the air separators. This tendency could be attributed to the fact that the performance depends primarily or only on the effect similar to the phenomena described by item (1) above.

\section{Accuracy in the Internal Flow Measurements}

The accuracy of the measurements of the overall fan flow rate is within $0.5 \%$ by the conventional Venturi-tube flowmeter and its ducting. The accuracy of pressure 
measurements by the 3-hole Pitot-tube is roughly $2 \%$ in an ideal two-dimensional flow condition. However, several macroscopic deviations, such as non-axi-symmetric structure of the fan rear parts and other factors, impair the overall accuracies.

A comparison of the fan flow rates measured by the Venturi-tube flowmeter and the integrated sectional flow rates used for preparation of Figs. 15 and 16 could provide an approximate measure of the accuracy (deviation) in the internal measurements. The procedure showed deviation levels of the downstream axial velocity $+15 \%$ for a larger flow, $+30 \%$ for the SW stalling equivalent region, $+70 \%$ for the SW deeply stalled equivalent region.

The reasons for the deviation are as follows; only one measuring position in the respective total circumference is possible, the fan structure is not axi-symmetric in the fan rear parts, a two-dimensional three-hole yawmeter is used in a three-dimensional and strong shear-flow environment, and the very low pressure of the fan is inconvenient for reliable measurements. All of the above could cause the deviation.

However, the main fan parts, the instrumentation and the method of measurement, and the procedure of conducting the experiments were the same for the SW condition and the AS condition. The authors are confident that this study provides a reliable comparison of the significant stall suppression effects by the AS device.

\section{Conclusion}

This experimental study revealed that application of an air separator drastically alters the internal flow structure of a fan from that in the original solid-wall condition. A comparative examination of the results, with attention to the stall suppression effects by the air separator, lead to the conclusion that the following effects are combined and integrated to contribute to the air separator performance. (1) Cleaning of the blade working environment by removal of low-energy flow around the blade tip, (2) separation and isolation of the reversed flow from the main flow, thus delaying the destabilization of the flow, (3) reinforcement of the inclinations of the meridional streamlines, thus increasing the fan Euler pressure for the decreasing fan flow rate, (4) stabilization of the flow structure by a blade-tip vortex ring through the air separator passage, and possibly, (5) enhancement of the axi-symmetry of the fan-tip flow field by the vortex ring and the strongly swirling tip flow. The last three are considered to be closely coupled with the tip-related flow structure that apparently dominates the stall suppression effect near the fan shut-off conditions, as suggested in the preceding study ${ }^{(1)}$ and (3).

\section{Acknowledgment}

The authors would like to express their deep thanks to the following students who have devoted their efforts to the research: Mr. Hiromi FUJIOKA, postgraduate student of 2008 in the Mechanical Engineering Department, Postgraduate School of Meisei University; and Mr. Shingo TANEI (2008), Mr. Katsumi HONDA and Mr. Takashi KUDO (2009), Mr. Hideyuki HAMABAYASI and Mr. Seishi FUJINAGA (2010), graduate students of Mechanical Engineering Department, Faculty of Sciences and Engineering, Meisei University.

\section{References}

(1) Yamaguchi, N., Ogata, M., and Kato, Y., Improvement of Stalling Characteristics of an Axial Fan by Radial-Vaned Air-Separators, 1st Report: Effects of Radial-Vaned Air Separators (in Japanese), Transaction of the Japan Society of Mechanical Engineers, Series $B$, Vol. 74, No. 746 (2008) , pp. 2163-2172

(2) Yamaguchi, N., Ogata, M., and Kato, Y., Improvement of Stalling Characteristics of an Axial Fan by Radial-Vaned Air-Separators, 2nd Report: Compact Radial-Vaned Air 
Separators (in Japanese), Transaction of the Japan Society of Mechanical Engineers, Series B, Vol. 74, No. 746 (2008), pp.2173-2181

(3) Yamaguchi, N., Ogata, M., and Kato, Y., Improvement of Stalling Characteristics of an Axial Fan by Radial-Vaned Air-Separators, Journal of Turbomachinery, Transaction of the American Society of Mechanical Engineers, Vol. 132, No. 2 (2010) , pp. 021015-1-10

(4) Yamaguchi, N., Ogata, M., and Kato, Y., Effects of Compact Radial-Vaned Air Separators on Stalling Characteristics of an Axial-Flow Fan, Journal of Turbomachinery, Transaction of the American Society of Mechanical Engineers, Vol. 132, No. 2, (2010-4), pp. 021009-1-8

(5) Yamaguchi, N., Casing Treatment (Review in Japanese), Journal of Turbomachinery Society of Japan, Vol.12, No.9 (1984), pp. 558-567

(6) Yamaguchi, N., Takami, I., and Arimura, H., Improvement of Air Separators of Axial Blowers (in Japanese), Proceedings of the 250th Kansai Branch Meeting of the Japan Society of Mechanical Engineers, (1991), pp.176-178

(7) Fujioka, Y., Yamaguchi, N. and Ogata, M., Internal Flow Patterns of an Axial Flow Fan Having Improved Stalling Characteristics Achieved by an Air Separator (in Japanese), Proceedings of 2008 Annual Meeting of the Japan Society of Mechanical Engineers, No. 08-1(2) (2008), pp. 59-60

(8) Yamaguchi, N. and Ogata, M., and Fujioka, Y., Internal Flow Patterns of an Axial Flow Fan Having Improved Stalling Characteristics Achieved by a Radial-Vaned Air Separator (in Japanese), Research Bulletin of Faculty of Science and Engineering, Meisei University, Japan, Vol. 45 (2009), pp. 21-28

(9) Tanaka, S., Yamaguchi, N. and Ogata, M., Internal Flow Patterns of an Axial Flow Fan Having Improved Stalling Characteristics Achieved by an Air Separator (in Japanese), Proceedings of 2009 Annual Meeting of the Japan Society of Mechanical Engineers, No. 09-1(2) (2009), pp. 53-54

(10) Yamaguchi, N. and Ogata, M., Internal Flow and Turbulence Conditions in an Axial Flow Fan Affected by an Air-Separator Device (in Japanese), Paper No. 1207, Proceedings of 2010 Meeting of Fluid Engineering Division, the Japan Society of Mechanical Engineers (2010), pp. 339-340 